\title{
Modeling automotive FlexRay transceivers for signal integrity and EMC simulations
}

\author{
H. Günther, U. Hilger, and S. Frei \\ Technische Universität Dortmund, Dortmund, Germany
}

\begin{abstract}
Automotive bus systems like FlexRay or CAN transmit safety critical data. To ensure correct functionality under all circumstances, extensive investigations about signal integrity and EMC have to be performed. To be able to use simulation in this validation process, suitable models for the components of the bus systems have to be developed. This paper shows how a combined transceiver model for signal integrity and EMC investigations can be created. The model shows good results in comparison to measurement data.
\end{abstract}

\section{Introduction}

Automotive bus systems are used to connect control units, intelligent sensors and actors in vehicles. From economical point of view extended cable networks are desirable. Systems are often operated close to their specification limits. To provide safety critical applications under these circumstances or electromagnetic disturbances, the functionality of a bus system must be ensured with sophisticated methods.

An approach to detect problematic behaviour and its causes is the computational investigation of the transmitted signal quality (signal integrity) of the physical layer of the bus system. Models for any component of a bus system have to be developed. Accuracy of models and methods has to be qualified by measurements with realistic systems.

To ensure a high immunity against electromagnetic disturbances, the transceivers should be qualified with special RF immunity tests. EMC simulations may help to investigate possibly critical cases. Therefore accurate EMC behavioural models are needed (Hilger et al., 2010).

This paper shows the capabilities of bus system signal integrity and EMC simulation and focuses on modelling of the transceiver device. SI transceiver models mainly deal

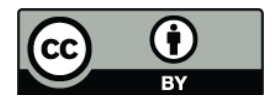

Correspondence to: $\mathrm{H}$. Günther (harald.guenther@tu-dortmund.de) with output characteristics while EMC models reflect the input behaviour of a device. However, intersections between those two fields exist and can be used to create a transceiver model to reflect both behaviours. As result the combined model is able to reflect the signal integrity behaviour of a real transceiver device in a wide range of input voltage and under electromagnetic disturbances and considers operation thresholds which limit normal operation ranges.

\section{Simulation models for SI and EMC}

\subsection{Signal integrity simulation model of FlexRay transceiver}

A FlexRay transceiver consists of general functional parts, e.g. for sending, receiving, and decoding data. Figure 1 shows a general structure of interconnection between those parts, which is the basis for the transceiver signal integrity simulation model (Günther et al., 2010).

For signal integrity investigations the most important part of the bus transceiver model is the output driver. The model is based on a physical approach which is shown schematically in Fig. 2. The three possible bus levels belonging to the internal transceiver states idle, active low and active high are realized by interconnection of two MOSFET transistors. They work as controlled switches based on the MOSFET equations and connect the bus output pin with ground or supply voltage, depending on the state of the transceiver. The adaption of the switching behavior and of the bus levels is done with a differentiable control function and the series connection of two diodes. When idle, both transistors are in high impedance mode and the transceiver is ready for signal reception. An additional ohmic voltage divider is used to model the correct impedance behavior in this case and provide the bias voltage. To ensure correct behavior in different load conditions and to model the slope correctly, capacitive effects of the transceiver device are modelled using output capacitors. 


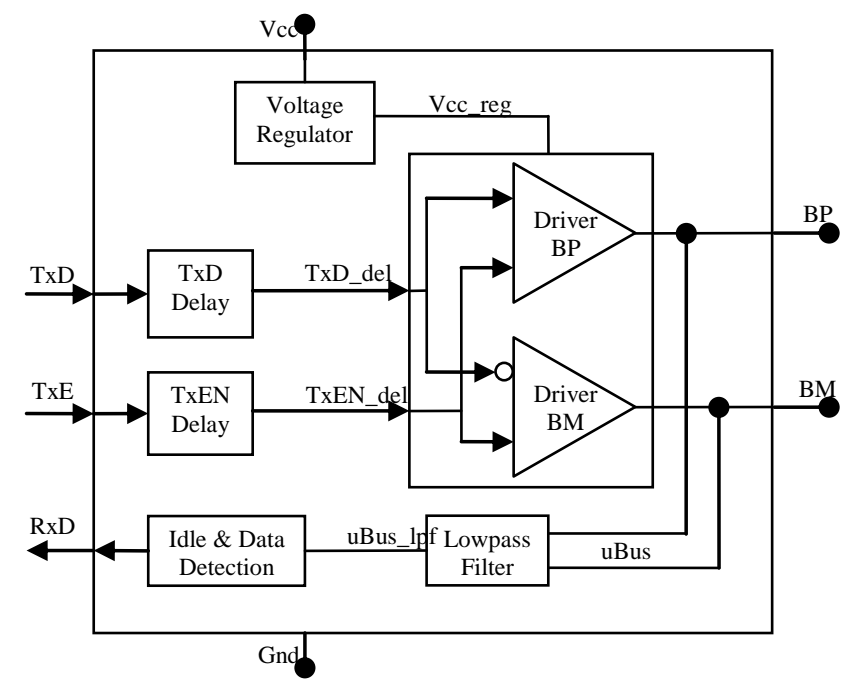

Fig. 1. Internal structure of output driver of FlexRay transceiver model.



Fig. 2. Schematic structure of output driver of FlexRay transceiver model.

With several parameters like switching voltages of the MOSFETs and diodes and parameters of the control function it is possible to match details of signal integrity behavior of the model with the behavior of the real transceiver device. Datasheet information and measurement data is used for this.

An advantage of this behavioral modeling approach compared to behavioral modeling approaches simply using measured characteristic curves (IBIS Open Forum, 2009) is the possibility to integrate for example temperature and supply voltage dependencies of the transceiver device in the model quite easily. By using basic physical equations the influences of several quantities can be integrated directly.

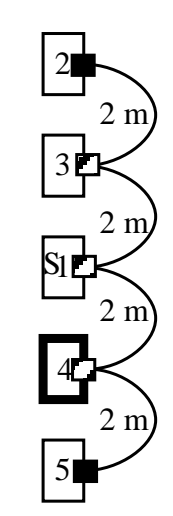

Topology A

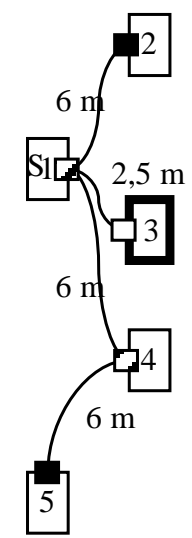

Topology B



Split Termination, Adapted

Split Termination, High Ohmic

Middle Termination (as displayed above)

Fig. 3. Structure of FlexRay topologies.



Fig. 4. Simulation and measurement results for topology A.

\subsection{Behavior of SI model in typical FlexRay networks}

The models for the bus system component were verified with measurement results for each component individually. The signal integrity behavior was validated at several load conditions. Good agreement between simulation and measurement data could be achieved.

Below the simulation results of two FlexRay bus system topologies shown in Fig. 3 are presented as example and compared with measurement data. It is shown that with the help of simulation based investigation conclusions about real world behavior of bus systems can be drawn.

Topology A is a chain connection of 5 bus nodes connected with bus lines with a length of $2 \mathrm{~m}$ each. Node 1 is sending. Figure 4 shows the comparison between simulation and measurement data at node 4 . The agreement between model behavior and measurement values is very good. 


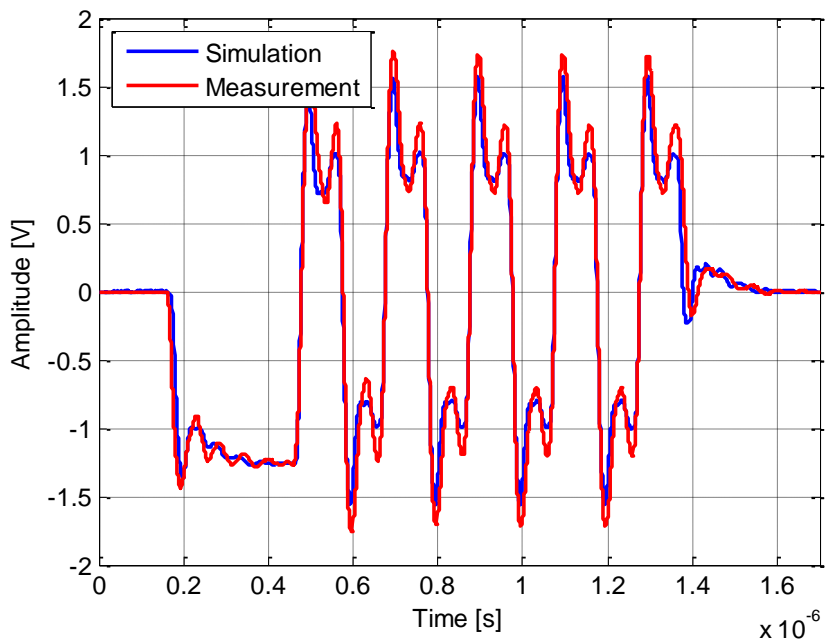

Fig. 5. Simulation and measurement results for topology B.

Topology B also includes 5 bus nodes connected with a network containing different line lengths. Node 1 is sending data onto the bus. Figure 5 shows the signal traces of simulation and measurement at node 3 . Again very good agreement between simulation and measurement data can be seen.

\subsection{EMC simulation model of FlexRay transceivers}

Because of the nonlinear behavior of the transceivers for EMC modeling, not only the determination of the stationary impedance is important, but also the dynamic behavior in case of bus communication failure (Hilger and Frei, 2008a,b). In other investigations the input impedance of IC's were mostly determined by the reflection measurements of the input pins and potential ESD protection elements were implemented as simple diode models (Boyer et al., 2007). The nonlinear effects near or over critical values are not considered in these examinations. In Hilger and Frei (2008b) investigations on modeling of CAN and FlexRay transceivers were made and very simple equivalent impedance models for EMC simulations were presented. However the behavior in case of transient disturbances or the partial destruction of bits by amplitude modulated signals in combination with ESD protection circuits and the transceiver internal signal processing is not considered. The receiver unit of a differential bus transceiver can be modeled as ideal high impedance comparator with low pass filter. High frequencies or fast transient pulses may not affect the correct detection of the signal and pulse width. In Table 1 the equivalent RC-circuits of investigated bus transceivers are shown.

Figure 6 shows a simple behavioral model of the bus receiver with equivalent impedance bus load, comparator and low pass filter.

The comparator detects and amplifies the differential bus voltage. The connected low pass will filter all high frequency

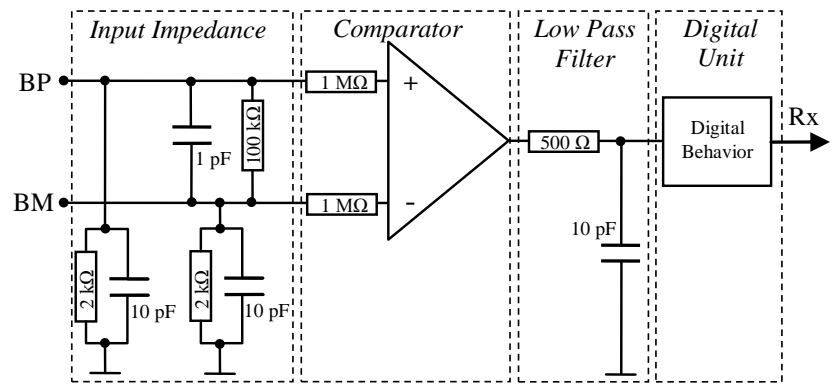

Fig. 6. Behavioral model of FlexRay receiver input.

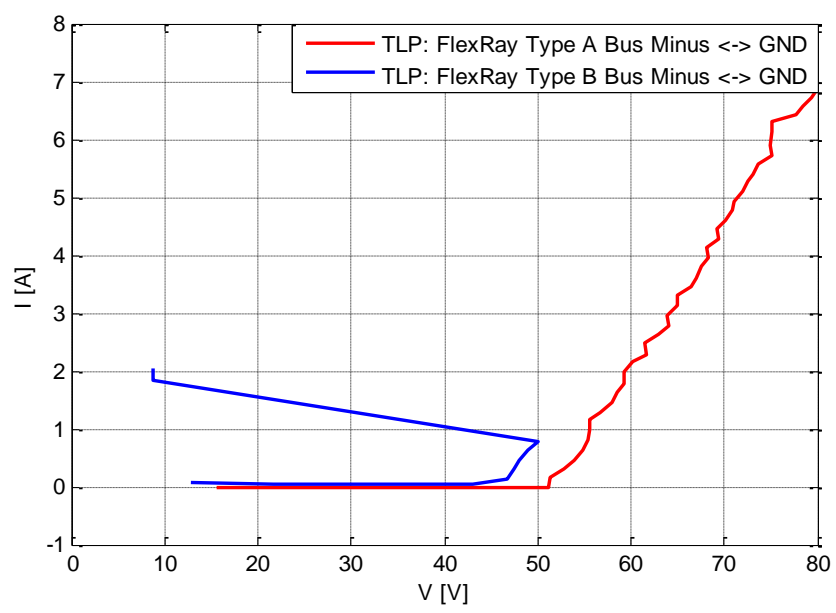

Fig. 7. TLP measurement, V/I characteristic of tested FlexRay transceivers.

parts of the signal and the digital unit is modeled as ideal $\mathrm{A} / \mathrm{D}-\mathrm{D} / \mathrm{A}$ converter.

In VHDL-AMS the transceiver behavior can be modeled as combination of discrete elements and variable input impedances (V/I- and f/Z-tables) which can be found by measuring the DC V/I characteristic and the impedance in failure point in frequency domain.

Figure 7 shows sample V/I-measurement results done with HPPI TLP (HPPI Transmission Line Pulser, 2010) of two typical FlexRay transceivers. At levels over 45-50 V internal ESD circuits may switch and the current increases critically. Below $45 \mathrm{~V}$ both transceivers have high impedances.

In Fig. 8 the frequency dependent critical failure voltages of the FlexRay transceivers are presented. Because of constructive variations and internal signal processing the transceivers have different immunity levels.

The voltage change between 90 and $100 \mathrm{MHz}$ are caused by resonances in the measurement setup. 
Table 1. Equivalent impedances of investigated bus drivers.

\begin{tabular}{lccc}
\hline Transceiver & CAN Type A & FlexRay Type A & FlexRay Type B \\
\hline Equivalent Impedance & $12 \mathrm{pF} \| 450 \Omega$ & $10 \mathrm{pF} \| 2 \mathrm{k} \Omega$ & $42 \mathrm{pF} \| 20 \mathrm{k} \Omega$ \\
\hline
\end{tabular}

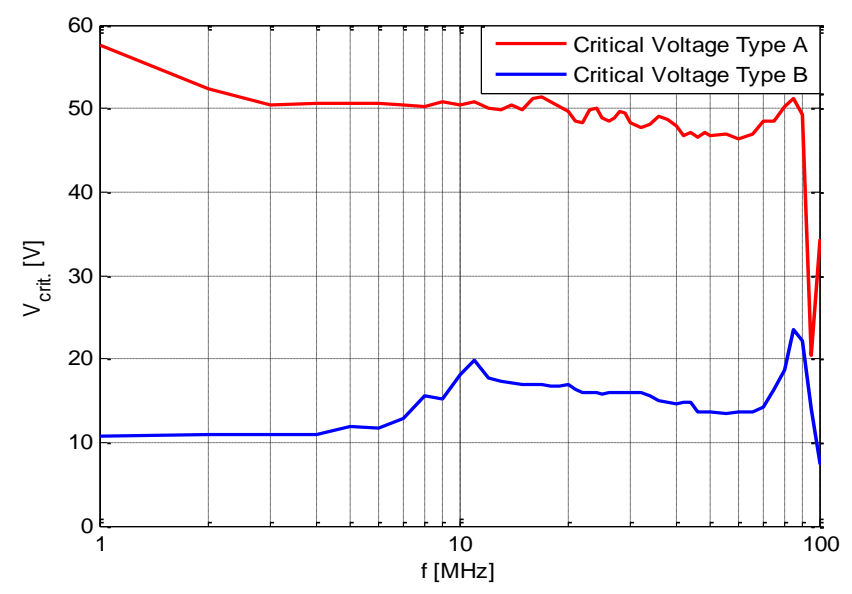

Fig. 8. Critical failure voltage of tested FlexRay transceivers.

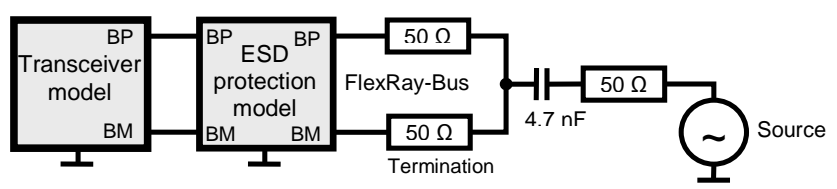

Fig. 9. Test setup incoupling with DPI method.

\subsection{Investigations of FlexRay transceivers in combina- tion with ESD protection circuits}

To perform EMC simulations in combination with ESD protection circuits for signal integrity investigations the transceiver models can be modularly extended with VHDLAMS models of protection elements.

ESD protection elements are intended for the protection against rarely occurring pulses. A permanent operation with CW-coupling above the breakdown voltage is not possible. The thermal power, generated by a coupled CW-current, can not be bypassed and will leads to destruction. The immunity simulations with sinusoidal disturbances were exemplarily done with a TVS diode array from Protek type GBLCS05C. The TVS array has a breakdown voltage of $6 \mathrm{~V} @ 1 \mathrm{~mA}$ and a parasitic impedance of $1.4 \mathrm{pF} \| 100 \mathrm{k} \Omega$.

According to the DPI method (IEC 62132-4:2006, 2006) the noise voltage will be coupled to both bus lines through the termination resistors and a capacitor. In Fig. 9 the simulation and measurement setup is shown.

A comparison between simulation and measurement is given in Fig. 10. The voltages are limited by the protec-
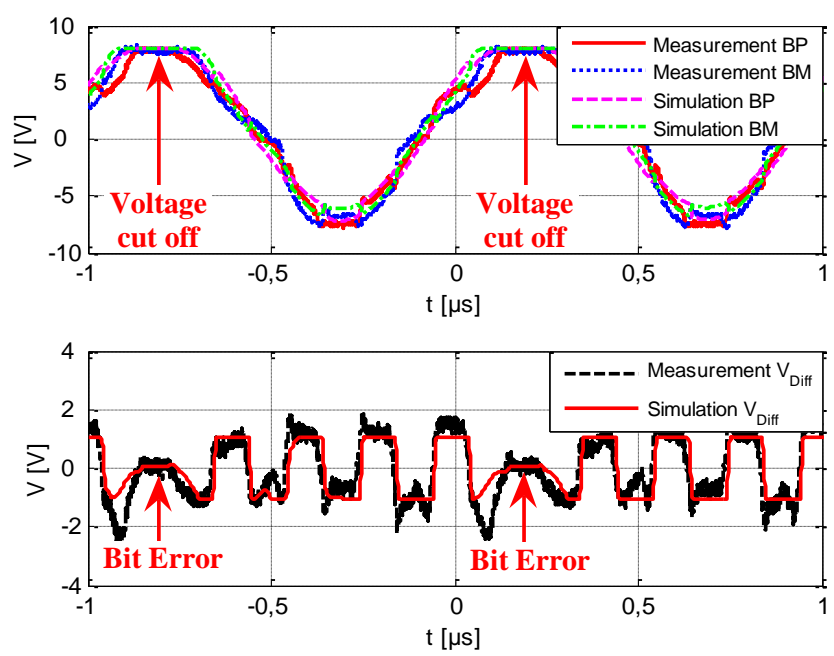

Fig. 10. Simulated and measured differential bus voltage, interference frequency $1 \mathrm{MHz}$ with FlexRay Type A and Protek TVS Array.

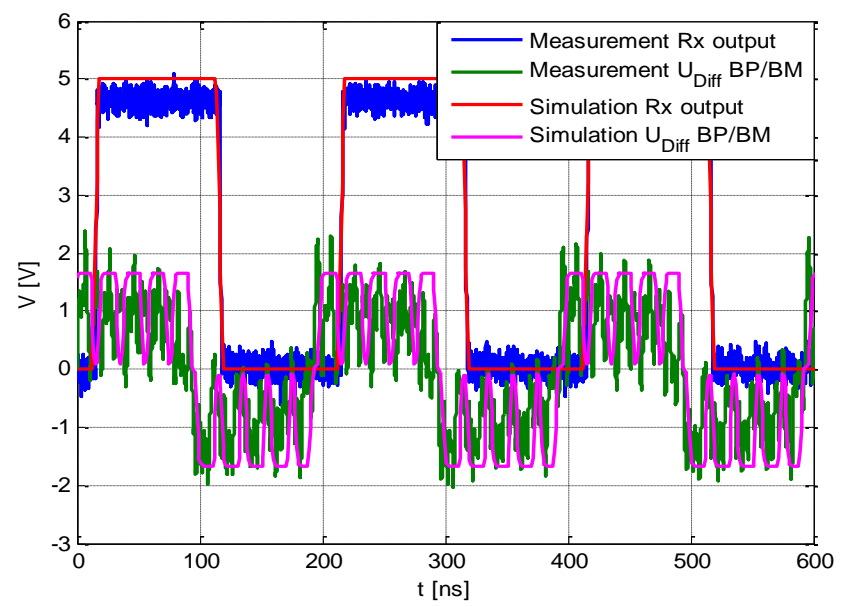

Fig. 11. Comparison between simulated and measured signal of a FlexRay transceiver.

tion element. At disturbance amplitude of about $8 \mathrm{~V}$, the transceiver can not detect the differential voltage anymore. The disturbed differential bus signal with the resulting bit errors can be seen below. The correlation between simulation and measurement results is very good.

To verify the low pass filter characteristics and the digital behavioral model in combination with ESD protection circuits measurements at high and low frequencies were done. 


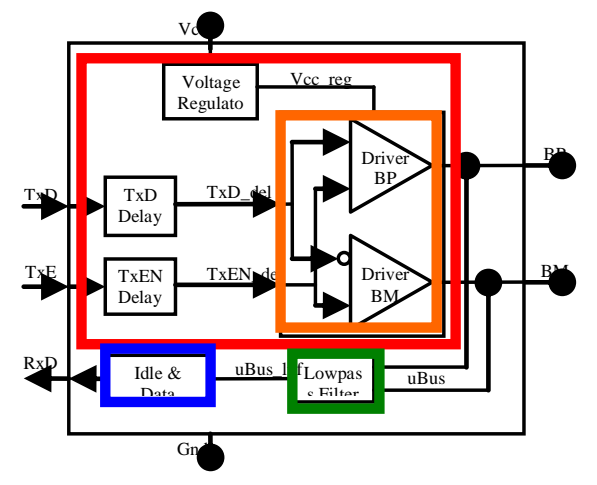

Structure of SI transceiver model

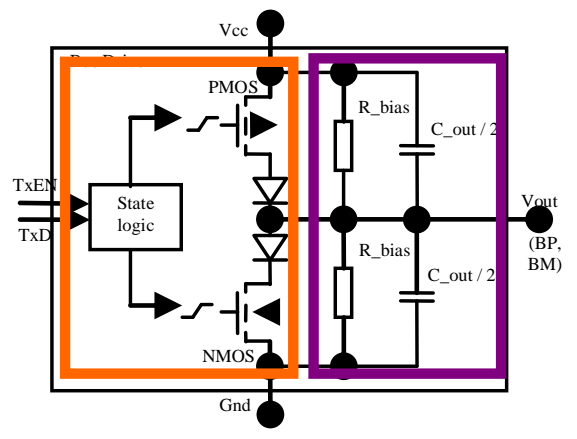

Structure of output driver

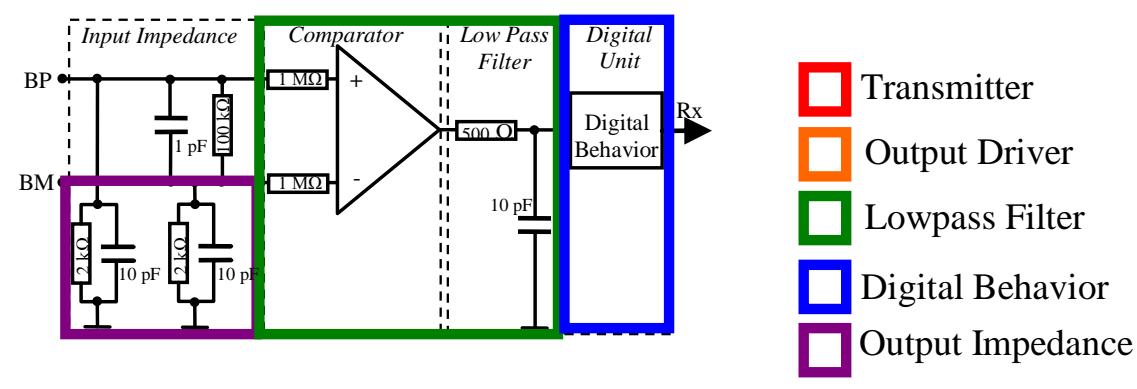

Structure of EMC transceiver model

Fig. 12. Common parts of SI and EMC transceiver model.

In Fig. 11 a comparison between the simulation with the behavioral model and a measured signal of the FlexRay transceiver can be seen. The transceiver is combined with the Protek TVS array. Interference frequency is $50 \mathrm{MHz}$ and the injected amplitude is $20 \mathrm{~V}$. The correlation of presented curves is good.

\section{A combined FlexRay transceiver model for SI and EMC simulations}

The presented SI model of the FlexRay transceiver mainly focuses on the output behavior of the bus driver, while the presented EMC model reflects the input behavior of the circuit. Integration of the two models to a combined SI and EMC transceiver simulation model is possible. Figure 12 gives an overview of the common parts of the two models.

The transmitting part with the output drivers is present only in the SI model, thus it will be used for the combined model. Lowpass filter, digital behavior, and output impedances are present in both SI and EMC models, but show big similarities in general parts. The EMC model contains additional information about differential impedances and operation limits.



Fig. 13. BCI test setup with passive star and four nodes.

\section{Simulation of BCI test with four node FlexRay network}

With a VHDL-AMS model of a BCI clamp from Hilger et al. (2010) it is possible to make virtual CAN or FlexRay network tests. In combination with a cable model (Zhang et al., 2008) and the transceiver models an overall simulation model can be created. To investigate an extended network 4 FlexRay nodes are interconnected with cable models of twisted pair cables. In Fig. 13 the BCI test setup is shown. According to FlexRay Protocol Specification (2005) all nodes are split terminated. Nodes 1 and 4 with two $1.3 \mathrm{k} \Omega$ resistors, nodes 2 and 3 with two $47 \Omega$ resistors and each 


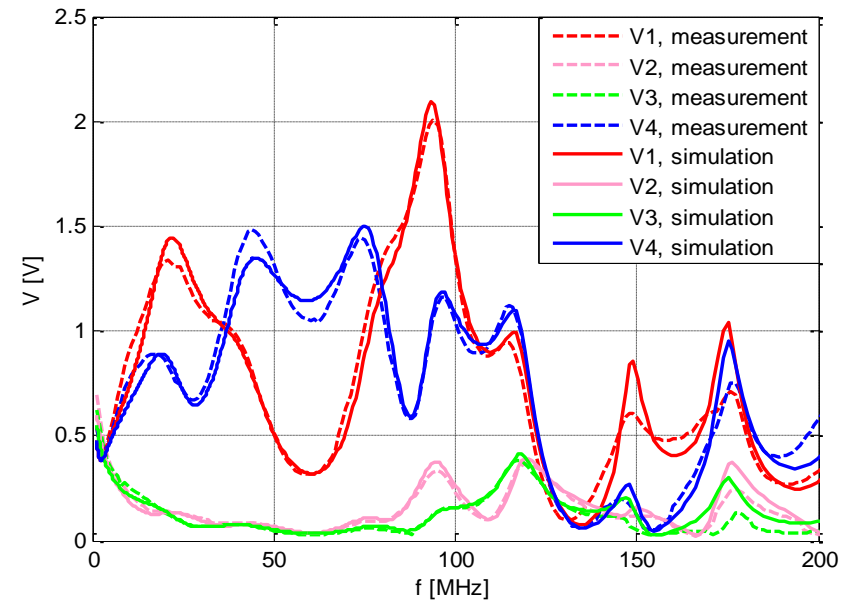

Fig. 14. Comparison between simulated and measured amplitudes at the four FlexRay nodes.

with $4.7 \mathrm{nF}$ to GND. Common mode chokes or other protection elements are not used in this setup. The BCI clamp is positioned on the cable of node 2 in a distance of $0.2 \mathrm{~m}$.

Figure 14 shows a comparison between the simulated and measured voltages at different FlexRay nodes. The measurements were done in the frequency domain with a network analyzer and in the time domain with a signal generator and oscilloscope. Here higher input power and active voltage probes were used. The BCI clamp was supplied with constant power of $10 \mathrm{dBm}$ over all frequencies. The parasitic effects of connectors, the PCB and discrete elements, like resistors and capacitors, were considered in the model. The model shows very good correlation up to $200 \mathrm{MHz}$ between simulated and measured results.

\section{Conclusion}

In order to investigate possible disturbances in bus system operation with simulation, behavioral models of bus transceivers for SI and EMC investigations were developed. SI model focus mainly on the output behavior of the transceivers, while EMC models reflect the input behavior. A detailed comparison of two developed models for these applications shows, that integration into a combined transceiver model for SI and EMC simulations is possible. Transmitter parts are taken from the SI model, since the EMC model gives detailed information about output impedances and operation limits. Digital receiving parts are present in both models. The combined model shows good matching with measurement results in an application example with disturbance signal injection through a BCI clamp.

\section{References}

Boyer, A., Bendhia, S., and Sicard, E.: Modeling of a Direct Power Injection Agression on a 16 Bit Microcontroler Input Buffer, EMC Compo 2007, 6th International Workshop on Electromagnetic Compatibility of Integrated Circuits, 2007.

FlexRay Protocol Specification: V2.1 Rev. A, http://www.flexray. com, 2005.

Günther, H., Frei, S., and Wenzel, T.: Simulation Methods for Signal Integrity of Automotive Bus Systems, APEMC, 2010.

Hilger, U. and Frei, S.: Modellierung von LIN-Transceivern für EMV-Simulationen im Kraftfahrzeug, EMV 2008, VDE Verlag, 2008a.

Hilger, U. and Frei, S.: Störfestigkeit von Bustransceivern gegen sinusförmiger Stögrößen in Verbindung mit nichtlinearen ESDSchutzelementen, EMV 2008, VDE Verlag, 2008b.

Hilger, U., Miropolsky, S., and Frei, S.: Modeling of Automotive Bus Transceivers and ESD-Protection Circuits for Immunity Simulations of Extended Networks, EMC Europe, Wroclaw, 2010.

HPPI Transmission Line Pulser: Type TLP3010, http://www.hppi. de, 2010.

IEC 62132-4:2006: Integrated circuits - Measurement of electromagnetic immunity $150 \mathrm{kHz}$ to $1 \mathrm{GHz}$ - Part 4: Direct RF power injection method, 2006.

IBIS Open Forum: I/O Buffer Information Specification, Online, available: http://www.eigroup.org/ibis, 2009.

Zhang, H., Siebert, K., Frei, S., Wenzel, T., and Mickisch, W.: Multiconductor Transmission Line Modeling with VHDL-AMS for EMC Applications, IEEE-EMC 2008 Symposium, Detroit, 2008. 